\title{
Expression of receptor interacting protein 3 and mixed lineage kinase domain-like protein-key proteins in necroptosis is upregulated in ulcerative colitis
}

\author{
Tielong Wu, Yuanyuan Dai, Lili Xue, Yingyue Sheng, Linlin Xu, Yuzheng Xue \\ Department of Gastroenterology, Affiliated Hospital of Jiangnan University, Wuxi 214062, China \\ Contributions: (I) Conception and design: T Wu, Y Dai; (II) Administrative support: All authors; (III) Provision of study materials or patients: All \\ authors; (IV) Collection and assembly of data: T Wu, Y Sheng; (V) Data analysis and interpretation: All authors; (VI) Manuscript writing: All authors; \\ (VII) Final approval of manuscript: All authors. \\ Correspondence to: Yuzheng Xue. Department of Gastroenterology, Affiliated Hospital of Jiangnan University, Wuxi 214062, China. \\ Email: 1480455860@qq.com.
}

Background: Ulcerative colitis (UC) is a chronic inflammatory colonic disease strongly associated with
intestinal epithelial cell (IEC) death. Necroptosis is characterized by a newly programmed cell death through
a caspase-independent pathway. Receptor interacting protein 3 (RIP3) and mixed lineage kinase domain-like
protein (MLKL) are very important in the pathway of necroptosis. However, their expression in UC remains
unelucidated. This study aimed to investigate the expression of RIP3 and MLKL in patients with UC, along
with its correlation with disease activity.

Methods: Colonic tissue samples were taken from 22 UC patients and 19 healthy controls. RIP3 and MLKL expression levels were evaluated by immunohistochemical staining and western blotting.

Results: RIP3 and MLKL were upregulated in inflamed tissues of $\mathrm{UC}(\mathrm{P}<0.01)$. No variations were observed in healthy control subjects and non-inflamed colons $(\mathrm{P}>0.05)$. RIP3 and MLKL were positively correlated with UC disease activity $(\mathrm{P}<0.01)$.

Conclusions: Our results suggest that necroptosis is strongly associated with intestinal inflammation in patients with UC. Further studies of necroptosis may be helpful in future treatments of UC.

Keywords: Ulcerative colitis (UC); receptor interacting protein 3 (RIP3); mixed lineage kinase domain-like protein (MLKL); intestinal inflammation

Submitted May 25, 2019. Accepted for publication Jun 26, 2019.

doi: 10.21037/apm.2019.07.04

View this article at: http://dx.doi.org/10.21037/apm.2019.07.04

\section{Introduction}

Ulcerative colitis (UC) is a chronic intestinal inflammatory disease characterized by chronic inflammation limited to the mucosal layer of the colon. It usually affects the rectum, and inflammation may extend throughout the entire colon (1). Pathologically, it is characterized by chronic inflammation and epithelial barrier damage (2). Intestinal epithelial cells (IECs) form the first intestinal barrier. Increasing evidence has shown that abnormal IEC death plays an important role in intestinal inflammation $(3,4)$.

Necrosis is a new caspase-independent mode of programmed cell death. Necroptosis is characterized by cell swelling, mitochondria dysfunction, plasma membrane permeabilization, and the release of cytoplasmic content to the extracellular space (5). Necroptosis can be activated by various stimuli such as lipopolysaccharides (LPS), physicalchemical stress, ionizing radiation, calcium overload, anticancer drugs, DNA damage, and other causes (6). Signaling can be initiated through the activation of members of the tumor necrosis factor (TNF) family, and it has been shown that this pathway is mediated by two kinases, receptorinteracting protein 1 (RIP1) and receptor-interacting protein 
Table 1 Clinicopathological characteristics of UC patients

\begin{tabular}{|c|c|}
\hline Number & Values $(n=22)$ \\
\hline Age (years), mean \pm SD [range] & $47 \pm 15[22-56]$ \\
\hline Gender (male/female) & $13 / 9$ \\
\hline \multicolumn{2}{|l|}{ Disease extent } \\
\hline Proctosigmoiditis & 7 \\
\hline Left-sided colitis & 10 \\
\hline Pancolitis & 5 \\
\hline \multicolumn{2}{|l|}{ Medication } \\
\hline 5-ASA & 17 \\
\hline Glucocorticoids & 3 \\
\hline Immunomodulators or biologics & 2 \\
\hline \multicolumn{2}{|l|}{ Smoking status } \\
\hline Smoking & 6 \\
\hline Non-smoking & 16 \\
\hline \multicolumn{2}{|l|}{ Clinical activity } \\
\hline Mild & 7 \\
\hline Moderate & 9 \\
\hline Severe & 6 \\
\hline \multicolumn{2}{|l|}{ Endoscopic Severity } \\
\hline Grade I & 6 \\
\hline Grade II & 6 \\
\hline Grade III & 7 \\
\hline Grade IV & 3 \\
\hline
\end{tabular}

ASA, aminosalicylic acid; UC, ulcerative colitis.

3 (RIP3) (7). RIP3 has been identified as a key mediator of necroptosis (8). When the protease activity of caspase- 8 is decreased, the necrosome that consists of RIP1 and RIP3 activates the pathway of necroptosis (9). Mixed lineage kinase domain-like protein (MLKL) is the main downstream component of RIP3 and RIP1 (10). Therefore, this study aimed to investigate the presence of RIP3 and MLKL in colonic mucosa in UC patients.

\section{Methods}

\section{Patients and disease activity}

Twenty-two patients with active UC and 19 healthy controls were enrolled into this study, which was conducted from December 2012 to November 2014 (Table 1). The diagnosis of UC patients was based on clinical symptoms, endoscopic findings, and histological signs, according to the Lennard-Jones criteria. Disease location was categorized using the Montréal classification system (11) [proctitis $(n=7)$, left-sided colitis $(n=11)$, and pancolitis $(n=4)]$. UC disease activity was measured by three indices, in which clinical activity was measured by the Mayo score (12), and endoscopic activity was measured by the Modified Baron Score (13). These activity scores were determined on the same day RIP3 and MLKL were measured.

\section{Materials}

Colonic biopsy specimens from patients with active UC included inflamed mucosa and non-inflamed mucosa. Control specimens were obtained from healthy volunteers during endoscopy. Two adjacent biopsies were taken from one spot: one for histology, which was immediately fixed in $10 \%$ neutral buffered formaldehyde; and another for RNA or protein analysis, which was snap-frozen in liquid nitrogen for protein extractions.

\section{Immunobistochemistry}

Colonic specimens were fixed in formalin and embedded in paraffin. Paraffin-embedded tissue sections $(2 \mu \mathrm{m})$ were dewaxed in dimethylbenzene solution and rehydrated in graded alcohols. Then, $1.5 \%$ of $\mathrm{H}_{2} \mathrm{O}_{2}$ was used to suppress endogenous tissue peroxidase activity. Antigen retrieval was conducted by boiling slides in $10 \mathrm{mM}$ of sodium citrate buffer. Subsequently, $3 \%$ of BSA was used to block nonspecific binding sites for $1 \mathrm{~h}$ at room temperature. Slides were incubated with rabbit anti-human antibody (RIP3: ab152130, MLKL: ab32125; Abcam, Cambridge, UK) at $4{ }^{\circ} \mathrm{C}$ overnight. Biotinylated anti-IgGs (Maixin, Fuzhou, China) were used as a secondary reagent. Next, slides were incubated with peroxidase-conjugated avidin-biotin complex. DAB (brown) peroxidase substrates were used to develop stains, and hematoxylin was used as a counterstain.

\section{Immunoblot analysis}

Western blotting analysis was performed as previously described (14). Fresh colon tissue samples were grounded into powder in liquid nitrogen, and a total of $20 \mu \mathrm{g}$ of total proteins were fractionated by SDS-PAGE. Proteins 
were transferred onto PVDF membranes, and membranes were blocked by $5 \%$ fat-free milk in Tris-buffered saline containing $0.1 \%$ Tween-20 for $1 \mathrm{~h}$ at room temperature. Anti-RIP3 (1:1,000; Abcam, Cambridge, UK), anti-MLKL (1:500, Sigma) and anti- $\beta$-actin (1:5,000; Sigma) antibodies were diluted in TBS-T and incubated overnight at $4{ }^{\circ} \mathrm{C}$. Then, the membranes were washed with horseradish peroxidase-conjugated rabbit anti-mouse IgG, and developed with ECL Plus. $\beta$-actin was used as a loading control. Densitometric analysis of the blots was performed using ImageJ software. Average integrated optical density was obtained by analyzing 5 fields of view for each slide evaluated by Image-Pro Plus version 5.0.

\section{Statistical analysis}

All statistical analysis was conducted using SPSS 22.0. For comparison between two groups, Student's $t$-test was used for normally distributed variables and Mann-Whitney U-test was used for non-normally distributed variables. For the comparison of more than two groups, ANOVA test was used for normally distributed variables while KruskalWallis test was used for non-normally distributed variables. Correlations among protein levels and disease activity were evaluated by Spearman's rank correlation coefficient (r) for non-parametric correlations. $\mathrm{P}<0.05$ was considered statistically significant.

\section{Ethics}

This study was approved by The Scientific Ethics Committee of Wuxi Third People's Hospital (No. 0228). All patients provided informed consent before participation, and this project abided the guidelines outline by the Declaration of Helsinki.

\section{Results}

\section{Expression levels of RIP 3 and MLKL were upregulated in inflamed tissues of UC patients}

Immunohistochemical staining was applied to determine the localization of RIP3 and MLKL in biopsies obtained from 22 patients with active UC and 19 healthy volunteers (controls). Immunohistochemistry revealed that RIP3- and MLKL-positive staining was mainly in inflamed tissues. Semi-quantitative analysis was performed to examine the expression of RIP3 and MLKL. This revealed that the expression of RIP3 and MLKL in IECs increased in inflamed tissues of UC patients, compared to noninflamed tissues and controls (Figure $1 A, B$ ). Average optical density (AOD) of RIP3 and MLKL was significantly higher in inflamed tissues compared to non-inflamed tissues and control groups (RIP3: AOD 0.1580 0.0639 vs. $0.1016 \pm 0.0442$ or $0.0979 \pm 0.0425$, MLKL: AOD $0.2060 \pm 0.0639$ vs. $0.1310 \pm 0.0570$ or $0.1230 \pm 0.0639 ; \mathrm{P}<0.01$ respectively) (Figure $1 C$ ).

Consistent with the expression levels assessed by immunohistochemical analyses, immunoblot analyses revealed that RIP3 and MLKL protein levels were elevated in inflamed tissues of UC patients compared with noninflamed tissues and controls (Figure 1D).

\section{Correlation of RIP3 and MLKL with disease activity}

The AODs of RIP3 and MLKL were higher in moderate and severe activity UC than in mild activity UC (RIP3: AOD $0.1742 \pm 0.0486$ vs. $0.1212 \pm 0.0469,0.2166 \pm 0.0519$ vs. $0.1212 \pm 0.0469$; MLKL: AOD $0.2127 \pm 0.0581$ vs. $0.1528 \pm 0.0405,0.2579 \pm 0.0503$ vs. $0.1528 \pm 0.0405$, respectively; $\mathrm{P}<0.01$ ) (Figure $2 A$ ). The AODs of RIP3 and MLKL in grades III-IV were also higher than in grades I and II (RIP3: AOD 0.2152 \pm 0.0424 vs. 0.1114 \pm 0.0454 , $0.2152 \pm 0.0424$ vs. $0.1492 \pm 0.0328$; MLKL: AOD $0.2458 \pm 0.0594$ vs. $0.1589 \pm 0.0448,0.2458 \pm 0.0594$ vs. $0.1867 \pm 0.0505$; respectively; $\mathrm{P}<0.05$ ) (Figure $2 B$ ). In addition, AODs of RIP3 and MLKL in UC-inflamed tissues were positively correlated with disease activity including clinical activity, colonoscopic activity, and histologic activity (Table 2), suggesting that RIP3 and MLKL levels are associated with UC disease activity. However, RIP3 and MLKL staining did not reveal any correlation with disease extent (Table 2).

\section{Discussion}

IECs form the first barrier between the inner environment and the lumen. In general, IEC death is restricted to the villous tip. Intestinal barrier function is disrupted when IEC death is increased and is diffusely distributed throughout the villous. Increased IEC death and intestinal inflammation represent two key features of UC. The major pathway of programmed cell death is apoptosis, which consists of the extrinsic pathway (triggered by the death receptor and ligand) and intrinsic pathway (controlled by the Bcl-2 family). Previous studies have shown that IECs were not sensitive to 
A

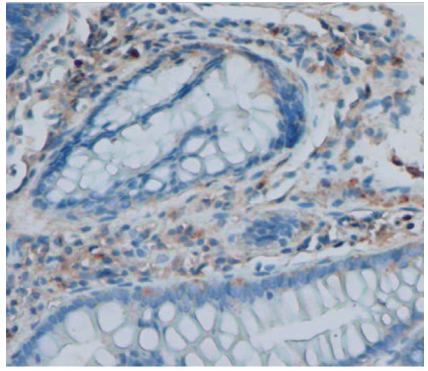

Normal

B

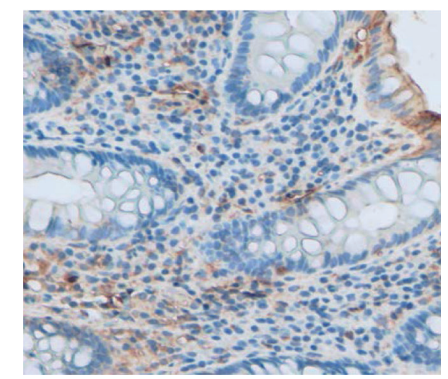

Normal

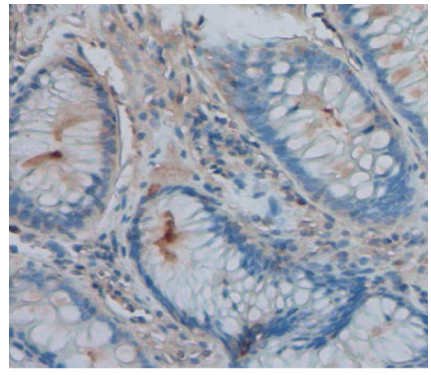

Non-inflamed UC

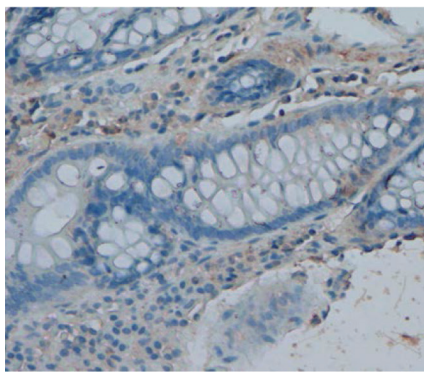

Non-inflamed UC

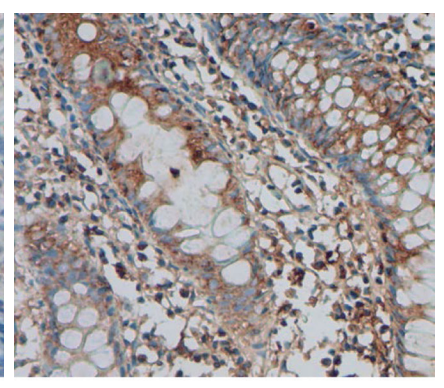

Inflamed UC

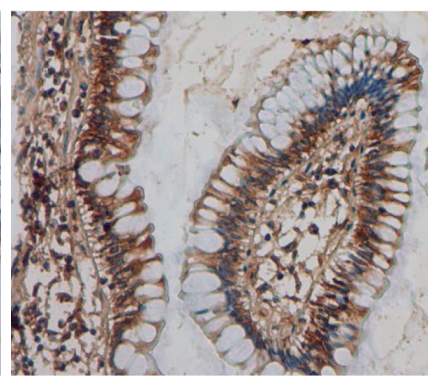

Inflamed UC
C
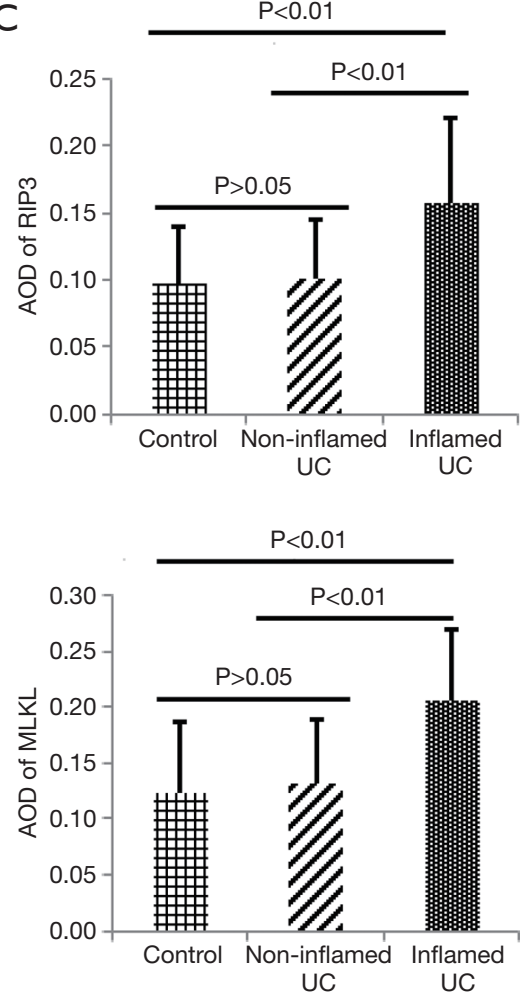

D
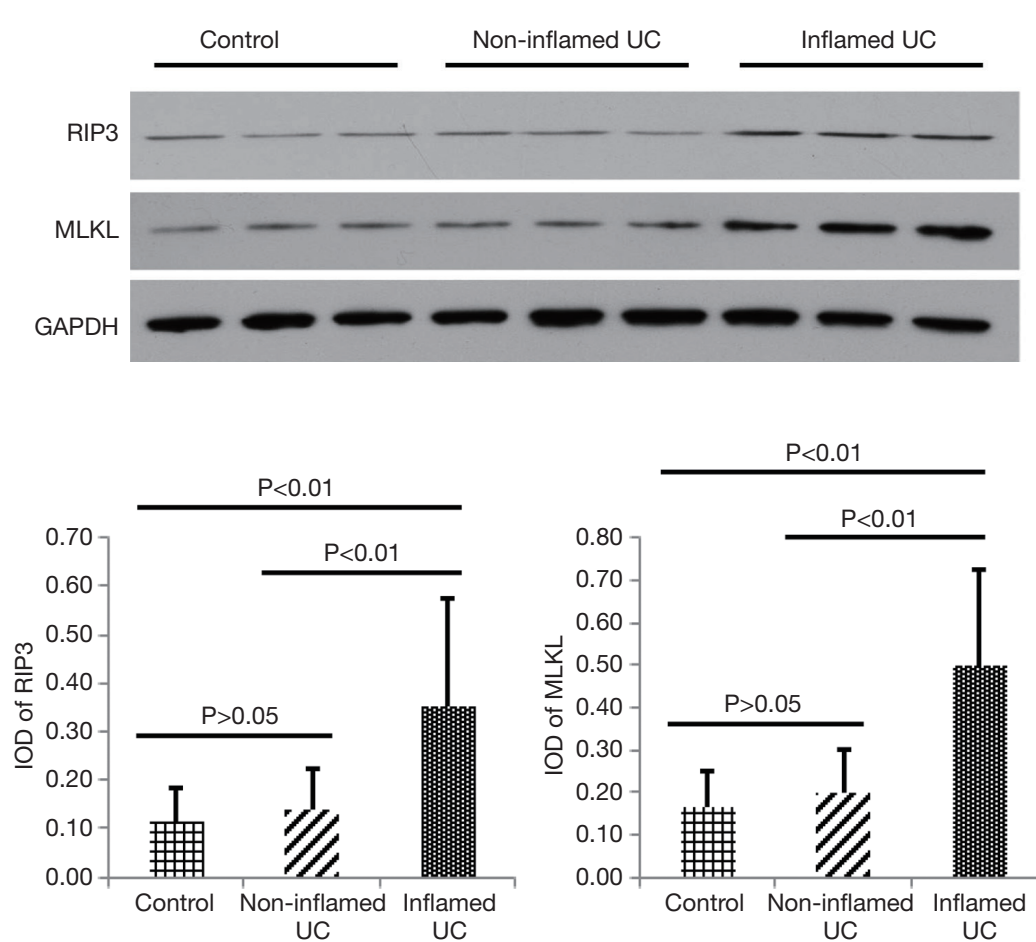

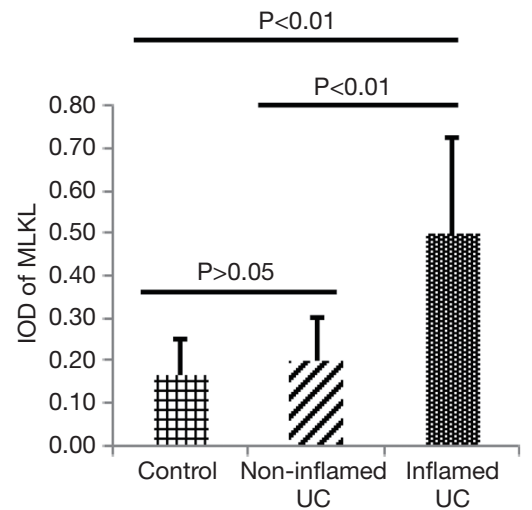

Figure 1 The expression of RIP3 and MLKL in intestinal epithelial cells increased in inflamed tissues of UC patients. (A) The expression, distribution and AOD of RIP3. (B) The expression, distribution and AOD of MLKL. Immunostaining of RIP3 and MLKL expression was robust in UC-inflamed colonic mucosa and low in UC-non-inflamed or normal colonic mucosa. The original magnifications were $\times 100$. (C) The AODs of RIP3 and MLKL were significantly higher in UC-inflamed colonic mucosa than in UC-non-inflamed or normal mucosa and the control group, $\mathrm{P}<0.01$. (D) Western blot analyses for RIP3 and MLKL. AOD, average optical density. 
Table 2 Correlation of RIP3 and MLKL expression with disease activity indicators in UC

\begin{tabular}{lcccc}
\hline \multirow{2}{*}{ Clinical parameters } & \multicolumn{2}{c}{ RIP3 } & & \multicolumn{1}{c}{ MLKL } \\
\cline { 2 - 5 } & $\mathrm{r}$ & $\mathrm{P}$ & $\mathrm{r}$ & 0.75 \\
Mayo score & 0.77 & $<0.01$ & 0.62 & $<0.01$ \\
Re-corrected Baron score & 0.81 & $<0.01$ & 0.19 & $>0.01$ \\
Disease extent & 0.04 & $>0.05$ & 0.05 \\
\hline
\end{tabular}

RIP3, receptor interacting protein 3; MLKL, mixed lineage kinase domain-like protein; UC, ulcerative colitis.

A

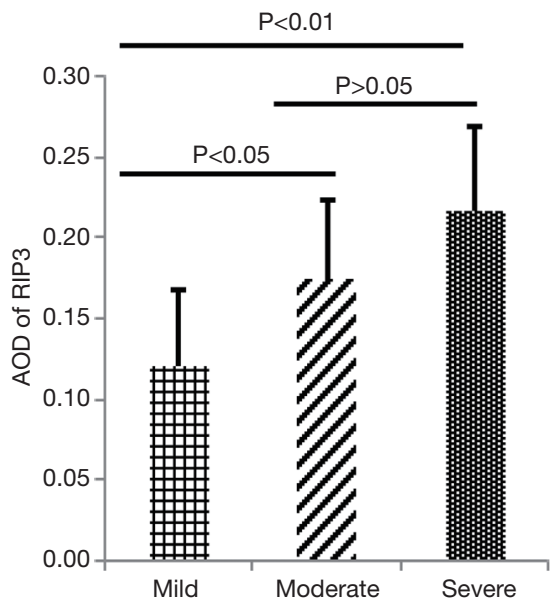

B

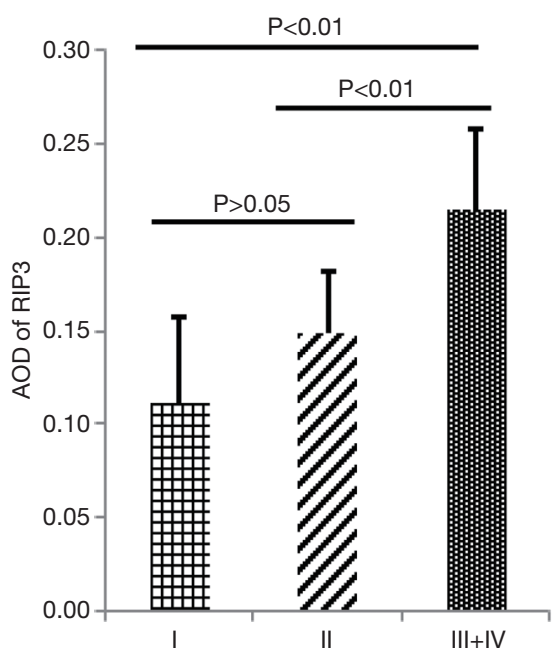

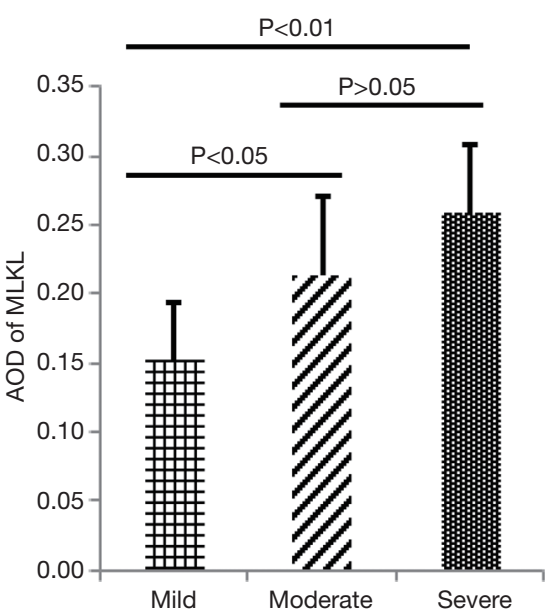

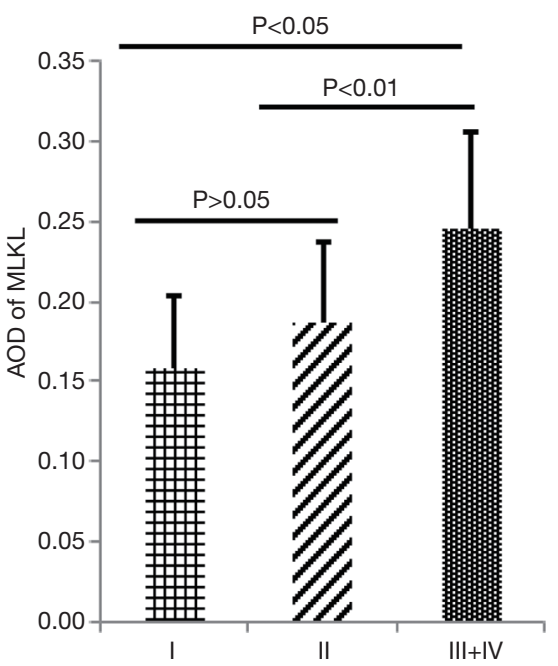

Figure 2 Expression levels of RIP3 and MLKL for clinical (12) and colonoscopic scores (13). (A) Clinical scores; (B) colonoscopic scores.

the extrinsic and intrinsic pathways (14-18). Therefore, it has been suggested that there may be another programmed death pathway involved in the dysfunction of IEC death.

Necroptosis, also called programmed necrosis, is similar to necrosis based on morphological features, but it is highly regulated by a series of intracellular signaling pathways. Recent studies have demonstrated that necroptosis plays an important role in various physiological and pathological disorders such as COPD (19), ischemia-reperfusion injury (20), neurodegenerative diseases (6), etc. Recent 
studies have suggested that necroptosis also participates in intestinal inflammation. Pierdomenico et al. (21) revealed that necroptosis was strongly associated with intestinal inflammation in children with inflammatory bowel disease (IBD) and contributed in heightening the inflammatory process. Günther et al. (22) found that necroptosis increased in the terminal ileum of patients with Crohn's disease. However, we wanted to investigate the expression of RIP3 and MLKL in UC patients and understand the relationship between necroptosis and disease activity.

Numerous studies reported that RIP3 and MLKL were key mediators in necroptosis through RIP3 interaction and MLKL phosphorylation-a process which promotes necroptosis $(10,23)$. Our study revealed that RIP3 and MLKL protein levels in inflamed tissues of UC patients were higher than in non-inflamed tissues and healthy control groups. However, we failed to find a significant difference between non-inflamed tissues of UC patients and tissue samples of healthy controls, which is in accordance with the results of the study conducted by Pierdomenico et al. (21). Moreover, RIP3 and MLKL levels in inflamed colonic tissue specimens of UC were significantly and positively correlated with UC disease activity including clinical activity (Mayo scores) and endoscopic activity (Modified Baron scores), indicating that RIP3 and MLKL may have important effects on intestinal inflammation.

However, this study has some limitations. First, this study was conducted with a relatively small number of subjects, which makes our results less conclusive. Second, the levels of caspase- 8 were not evaluated; thus, the relationship between caspase- 8 and necroptosis proteins in the inflamed colon could not be confirmed. Finally, the contribution of RIP3 and MLKL to the intestinal inflammatory process was not determined.

\section{Conclusions}

In conclusion, we demonstrated that RIP3 and MLKL were upregulated in UC, and that it correlated positively with disease activity. Our results indicate that necroptosis may be involved in intestinal inflammation. However, the role of necroptosis in disease treatment and prognosis remains to be determined. Compared to other cell death pathways such as apoptosis and necrosis, the signal mechanism of the necroptosis pathway is not completely understood. Further studies are needed to clarify the role of necroptosis in the pathogenesis of $\mathrm{UC}$, as well as its possible implications in treatment.

\section{Acknowledgments}

Funding: Our research was supported by the Foundation for "Talents in Six Fields" of the Jiangsu Province (WSN066), the Social Development Project of Wuxi City (CSE31N1415), the Wuxi City Health and Family Planning Commission "Science and Education Strong" Youth Talent Project (QNRC096), the Wuxi City Health and Family Planning Commission "Science and Education Strong" Key Talent Project (ZDRC039), and the Wuxi City Health and Family Planning Commission Youth Research Project (Q201747).

\section{Footnote}

Conflicts of Interest: The authors have no conflicts of interest to declare.

Ethical Statement: The authors are accountable for all aspects of the work in ensuring that questions related to the accuracy or integrity of any part of the work are appropriately investigated and resolved. This study was approved by The Scientific Ethics Committee of Wuxi Third People's Hospital (No. 0228) and written informed consent was obtained from all patients.

\section{References}

1. Kornbluth A, Sachar DB, Practice Parameters Committee of the American College of Gastroenterology. Ulcerative colitis practice guidelines in adults: American College Of Gastroenterology, Practice Parameters Committee. Am J Gastroenterol 2010;105:501-23.

2. Johnson DH, Khanna S, Smyrk TC, et al. Detection rate and outcome of colonic serrated epithelial changes in patients with ulcerative colitis or Crohn's colitis. Aliment Pharmacol Ther 2014;39:1408-17.

3. Chen L, Park SM, Turner JR, et al. Cell death in the colonic epithelium during inflammatory bowel diseases: CD95/Fas and beyond. Inflamm Bowel Dis 2010;16:1071-6.

4. Sträter J, Wellisch I, Riedl S, et al. CD95 (APO-1/Fas)mediated apoptosis in colon epithelial cells: a possible role in ulcerative colitis. Gastroenterology 1997;113:160-7.

5. Giampietri C, Starace D, Petrungaro S, et al. Necroptosis: molecular signalling and translational implications. Int J Cell Biol 2014;2014:490275.

6. Liu S, Wang X, Li Y, et al. Necroptosis mediates TNFinduced toxicity of hippocampal neurons. Biomed Res Int 
2014;2014:290182.

7. Zhang N, Chen Y, Jiang R, et al. PARP and RIP1 are required for autophagy induced by 11 '-deoxyverticillin $\mathrm{A}$, which precedes caspase-dependent apoptosis. Autophagy 2011;7:598-612.

8. Zhang DW, Shao J, Lin J, et al. RIP3, an energy metabolism regulator that switches TNF-induced cell death from apoptosis to necrosis. Science 2009;325:332-6.

9. Kikuchi M, Kuroki S, Kayama M, et al. Protease activity of procaspase- 8 is essential for cell survival by inhibiting both apoptotic and nonapoptotic cell death dependent on receptor-interacting protein kinase 1 (RIP1) and RIP3. J Biol Chem 2012;287:41165-73.

10. Zhao J, Jitkaew S, Cai Z, et al. Mixed lineage kinase domain-like is a key receptor interacting protein 3 downstream component of TNF-induced necrosis. Proc Natl Acad Sci U S A 2012;109:5322-7.

11. Silverberg MS, Satsangi J, Ahmad T, et al. Toward an integrated clinical, molecular and serological classification of inflammatory bowel disease: report of a Working Party of the 2005 Montreal World Congress of Gastroenterology. Can J Gastroenterol 2005;19 Suppl A:5A-36A.

12. Walmsley RS, Ayres RC, Pounder RE, et al. A simple clinical colitis activity index. Gut 1998;43:29-32.

13. Feagan BG, Greenberg GR, Wild G, et al. Treatment of ulcerative colitis with a humanized antibody to the alpha4beta7 integrin. N Engl J Med 2005;352:2499-507.

14. Iimura M, Nakamura T, Shinozaki S, et al. Bax is downregulated in inflamed colonic mucosa of ulcerative colitis. Gut 2000;47:228-35.

Cite this article as: Wu T, Dai Y, Xue L, Sheng Y, Xu L, Xue Y. Expression of receptor interacting protein 3 and mixed lineage kinase domain-like protein-key proteins in necroptosis is upregulated in ulcerative colitis. Ann Palliat Med 2019;8(4):483489. doi: 10.21037/apm.2019.07.04
15. Seidelin JB, Nielsen OH. Epithelial apoptosis: cause or consequence of ulcerative colitis. Scand J Gastroenterol 2009;44:1429-34.

16. Lu J, Caplan MS, Saraf AP, et al. Platelet-activating factorinduced apoptosis is blocked by Bcl-2 in rat intestinal epithelial cells. Am J Physiol Gastrointest Liver Physiol 2004;286:G340-50.

17. Souza HS, Tortori CJ, Castelo-Branco MT, et al. Apoptosis in the intestinal mucosa of patients with inflammatory bowel disease: evidence of altered expression of FasL and perforin cytotoxic pathways. Int J Colorectal Dis 2005;20:277-86.

18. Karamanolis DG, Kyrlagkitsis I, Konstantinou K, et al. The Bcl-2/Bax system and apoptosis in ulcerative colitis. Hepatogastroenterology 2007;54:1085-8.

19. Mizumura K, Cloonan SM, Nakahira K, et al. Mitophagydependent necroptosis contributes to the pathogenesis of COPD. J Clin Invest 2014;124:3987-4003.

20. Gao S, Andreeva K, Cooper NG. Ischemia-reperfusion injury of the retina is linked to necroptosis via the ERK1/2-RIP3 pathway. Mol Vis 2014;20:1374-87.

21. Pierdomenico M, Negroni A, Stronati L, et al. Necroptosis is active in children with inflammatory bowel disease and contributes to heighten intestinal inflammation. Am J Gastroenterol 2014;109:279-87.

22. Günther C, Martini E, Wittkopf N, et al. Caspase-8 regulates TNF- $\alpha$-induced epithelial necroptosis and terminal ileitis. Nature 2011;477:335-9.

23. Sun L, Wang $H$, Wang $Z$, et al. Mixed lineage kinase domain-like protein mediates necrosis signaling downstream of RIP3 kinase. Cell 2012;148:213-27. 